\title{
Fatores associados ao baixo peso ao nascer em crianças do Rio Grande do Sul
}

Factors associated to low birth weight in children from Rio Grande do Sul

\author{
Angelica Peripolli', Anaelena Bragança de Moraes", \\ Luciane Flores Jacobi"', Roselaine Ruviaro Zanini'
}

\begin{abstract}
RESUMO
Neste estudo, foram identificados e quantificados os fatores de risco associados aos nascidos vivos com baixo peso no Rio Grande do Sul, em 2011, utilizando dados do Sistema de Informações de Nascidos Vivos. A prevalência de baixo peso ao nascer (BPN) foi de 8,1\%. Por meio do ajuste por regressão logística, foram propostos dois modelos. Para o primeiro, as variáveis preditoras de risco e significativas a $5 \%$ foram: mães com 35 anos ou mais; nulíparas; com ensino fundamental II ou ensino médio; separadas judicialmente ou divorciadas; mães que não fizeram exame pré-natal; nascimento ocorrido fora do hospital; prematuridade; sexo feminino e que apresentaram anomalia congênita. As variáveis de efeito protetor foram: mães adolescentes e que não trabalhavam fora de casa. $O$ tipo de parto foi incluído no segundo modelo proposto, o qual foi mais apropriado para estimar a probabilidade de um nascido vivo nascer com baixo peso. Conclui-se que é importante considerar ações efetivas aos grupos vulneráveis, no sentido de redução das taxas de BPN, tendo em vista a forte associação com as taxas de mortalidade infantil.
\end{abstract}

Palavras-chave: Regressão logística múltipla; Fatores de risco; Baixo peso ao nascer; SINASC.

\begin{abstract}
In this study were identified and quantified the risk factors associated to live births with low birth weight in Rio Grande do Sul, in 2011 using data from the Information System on Live Births. The prevalence of low birth weight (LBW) was 8.1\%. By adjusting logistic regression, two models were proposed. For the first one, the predictors significant risk variables at $5 \%$ were: mothers aged 35 or more; nulliparous; with elementary school II or high school; legally separated or divorced; mothers who did not receive prenatal care; birth that occurred outside the hospital; prematurity; female and who had congenital anomaly. The protective effects variables were teenage mothers and who did not work outside the home. The type of delivery was included in the second model proposed, which was more appropriate to estimate the probability of a live birth born underweight. It was possible to conclude that it is important to consider effective actions to vulnerable groups aiming to reduce LBW rates, examining the strong association with infant mortality rates.
\end{abstract}

Keywords: Multiple logistic regression; Risk factors; Low birth weight; SINASC.

\footnotetext{
' Universidade Federal de Santa Maria, Santa Maria, Brasil. E-mail: angelicaperipolli@gmail.com.

" Universidade Federal de Santa Maria, Santa Maria, Brasil. E-mail: anaelena@smail.ufsm.br.

III Universidade Federal de Santa Maria, Santa Maria, Brasil. E-mail: lucianefj8@gmail.com.

IV Universidade Federal de Santa Maria, Santa Maria, Brasil. E-mail: rrzanini63@gmail.com.
} 


\section{INTRODUÇÃO}

O peso ao nascer é o primeiro peso do feto ou recém-nascido (RN), obtido após o nascimento (WORLD HEALTH ORGANIZATION, 2010), sendo o baixo peso ao nascer (BPN), definido pela Organização Mundial da Saúde (OMS), como sendo o peso ao nascer de um nascido vivo (NV) inferior a 2.500 gramas. Esse ponto de corte, adotado como parâmetro internacional, baseia-se em observações epidemiológicas de que crianças pesando menos do que $2.500 \mathrm{~g}$ têm, aproximadamente, 20 vezes mais risco de morrer do que bebês com mais peso (KRAMER, 1987).

De acordo com McCormick (1985), o peso de nascimento é o fator isolado mais importante na determinação da sobrevivência infantil, pois crianças com baixo peso apresentam um risco muitas vezes maior de morrer ou adoecer no primeiro ano de vida. Por isso, o BPN tem sido destaque em vários estudos epidemiológicos com o objetivo de identificar e estimar os fatores de risco, na tentativa de elaborar intervenções que possam reduzi-los, prevenindo a ocorrência dos mesmos.

A importância do BPN para a saúde pública é determinada não apenas pelos riscos de mortalidade e morbidade do RN, mas também pela frequência com que este ocorre. As maiores prevalências de BPN são observadas nos países em desenvolvimento, como uma consequência das piores condições de vida destes locais (HORTA; BARROS; HALPERN; VICTORA, 1996). Apesar dos grandes avanços na área de cuidados com o RN de risco, o nascimento de crianças com BPN é um dos grandes desafios para os serviços de saúde pública. As políticas e práticas em saúde pública para a redução do risco são implantadas a partir do conhecimento dos fatores de risco associados (TIAGO; CALDEIRA; VIEIRA, 2008). As informações sobre os nascidos vivos (NV) são encontradas na Declaração de Nascido Vivo (DN), um documento preenchido nos hospitais e instituído em 1990 pelo Ministério da Saúde, com informações de caráter demográfico, epidemiológico e clínico da gestante, do parto e do RN, com o objetivo de reunir informações epidemiológicas referentes aos nascimentos informados em todo o território nacional. 
Por intermédio desses registros é possível subsidiar as intervenções relacionadas à saúde da mulher e da criança, bem como ações de atenção à gestante e ao RN (MINISTÉRIO DA SAÚDE, 2009).

Dada a importância do baixo peso para os desfechos em saúde, este estudo tem como objetivo principal identificar e estimar os fatores de risco associados à ocorrência de baixo peso em nascidos vivos no RS, em 2011, além de realizar inferências epidemiológicas.

\section{MATERIAIS E MÉTODOS}

Foram utilizados dados de NV do Rio Grande do Sul no ano de 2011, que provieram das DN's que constam no Sistema de Nascidos Vivos (SINASC). Estes dados foram obtidos no site do Departamento de Informações do Sistema Único de Saúde (DATASUS), que fornece informações tanto da mãe como do seu bebê, por meio do programa TabWin32.

Primeiramente, foram identificados códigos errados ou dados faltantes nos registros. Foram considerados somente os bebês provenientes de gestação única, pois a probabilidade de neonatos de gravidez múltipla serem pequenos para a idade gestacional (PIG) é maior, porque a taxa de crescimento fetal diminui em virtude da influência de falta de espaço disponível no útero materno (HARRISON; WEINER; TANNER, 1971). A variável dicotômica, nascer ou não com baixo peso, foi considerada como o desfecho, sendo excluídos do estudo os bebês que não apresentaram a informação sobre o peso ao nascer ou que tinham peso inferior a 500g, o que representou 3.388 NV, totalizando 134.322 NV de gestação única.

As covariáveis foram recodificadas: idade da mãe em anos (20 a 34, $\geq 35$ e < 20); paridade (sem filhos, de 1 a 2 e > 3); escolaridade (superior incompleto ou completo, ensino fundamental II ou ensino médio, sem escolaridade ou ensino fundamental I); estado civil (casada ou união estável, solteira, viúva e separada judicialmente ou divorciada); ocupação (do lar, outra); número de consultas pré-natais (nenhuma, de 1 a 6, 27); tipo de parto (vaginal, cesáreo); local de ocorrência do nascimento (hospital, outro) e duração da gestação em semanas $(<37, \geq 37)$. 
As referentes ao nascido vivo foram: sexo (masculino, feminino); cor da pele (branca, preta, amarela, parda ou indígena) e anomalia congênita (sem, com). Para o ajuste dos modelos foi utilizada regressão logística múltipla a qual permite análise do efeito de uma ou mais variáveis independentes (discretas ou contínuas) sobre uma variável dependente dicotômica. E, assim, pode-se estimar a probabilidade da ocorrência do desfecho em função dos fatores significativos (HOSMER; LEMESHOW, 1989).

A equação de regressão é dada por (1):

$$
\ln \left[\frac{p}{1-p}\right]=\ln [o d d s]=\beta_{0}+\beta_{1} x_{1}+\beta_{2} x_{2}+\ldots+\beta_{p} x_{p}
$$

onde:

ln =logaritmo neperiano,

$\beta_{\mathrm{i}}=$ coeficientes e

$x_{i}$ variáveis preditoras.

A equação da probabilidade é dada por (2):

$$
P=\frac{1}{1+e^{-\left(\beta_{0}+\beta_{1} x_{1}+\beta_{2} x_{2}+\ldots+\beta_{p} x_{p}\right)}}=\frac{1}{1+e^{-(\operatorname{lnodds})}}
$$

Em Epidemiologia, a pesquisa causal requer que duas distinções fundamentais sejam realizadas: entre aqueles que realmente têm e aqueles que não têm os fatores de risco que estão sendo estudados (variável independente); e entre aqueles que realmente têm e os que não têm a doença a ser estudada (variável dependente) (JEKEL; ELMORE; KATZ, 1999).

Dentre as medidas de associação tem-se: a odds que é a razão entre o número de eventos observados e o número de eventos não observados, desta forma a odds é utilizada para designar chances a favor versus contra; e a odds ratio (OR) ou razão de 
chances, que é definida como a chance da doença entre os indivíduos expostos dividida pela chance da doença entre os indivíduos não expostos.

$\mathrm{Na}$ análise de regressão logística simples, cada variável candidata a ser avaliada como possível fator de risco independente para o baixo peso foi incluída, individualmente, nos modelos. As variáveis com significância estatística ( $p$-valor $\leq 25 \%$ ) foram incluídas nos modelos multivariáveis e, os respectivos coeficientes foram avaliados, conjuntamente, por meio do teste de Wald (HOSMER; LEMESHOW, 1989).

Após o ajuste dos modelos pelo teste de Hosmer-Lemeshow, foi obtido o percentual global de capacidade de classificação do modelo. Além da avaliação e a análise dos resíduos pela distância de $\mathrm{D}$ de Cook para verificar a presença de observações influentes (leverage) e dos resíduos estudentizados, para identificar possíveis outliers. As análises foram realizadas utilizando o programa estatístico PASW 18.0.

\section{RESULTADOS}

Considerando os 134.322 NV de gestação única no RS, observou-se que 10.879 NV (8,1\%) apresentaram BPN, sendo a média 3.184,28g (Desvio-padrão=536,489g), com mínimo de 500g e o máximo de 6.860g.

Na Tabela 1 são apresentados os fatores de risco para o BPN provenientes da análise de regressão simples e os valores do OR Bruto e dos Intervalos de Confiança (95\%).

Tabela 1 - Distribuição de frequências e resultados da análise de regressão logística simples para os NV, tendo como desfecho o BPN, SINASC/RS, 2011

\begin{tabular}{|c|c|c|c|c|c|}
\hline \multirow{2}{*}{$\begin{array}{l}\text { Variáveis } \\
\text { independentes }\end{array}$} & \multirow{2}{*}{ Total (\%) } & \multicolumn{2}{|c|}{ BPN (\%) } & \multirow{2}{*}{ OR Bruto (IC 95\%) } & \multirow{2}{*}{$\mathrm{p}$-valor } \\
\hline & & Sim & Não & & \\
\hline \multicolumn{6}{|l|}{ Idade materna (anos) } \\
\hline 20 a 34 & $93.518(69,6)$ & $7.139(7,6)$ & $86.379(92,4)$ & 1 & \\
\hline$\geq 35$ & $19.129(14,2)$ & $1.783(9,3)$ & $17.346(90,7)$ & $1,24(1,18-1,31)$ & $<0,001$ \\
\hline$<20$ & $21.675(16,1)$ & $1.957(9,0)$ & $19.718(91,0)$ & $1,20(1,14-1,27)$ & $<0,001$ \\
\hline \multicolumn{6}{|l|}{ Paridade } \\
\hline 1 a 2 & $55.410(43,9)$ & $3.808(6,9)$ & $51.602(93,1)$ & 1 & \\
\hline Nulípara & $54.646(43,3)$ & $4.892(9,0)$ & $49.754(91,0)$ & $1,33(1,28-1,39)$ & $<0,001$ \\
\hline$\geq 3$ & $16.233(12,9)$ & $1.541(9,5)$ & $14.692(90,5)$ & $1,42(1,34-1,51)$ & $<0,001$ \\
\hline \multicolumn{6}{|l|}{ Escolaridade* } \\
\hline Superior Inc./Comp. & $93.021(69,8)$ & $7.032(7,6)$ & $85.989(92,4)$ & 1 & \\
\hline Fund. II/Ens. Médio & $39.930(29,9)$ & $3.696(9,3)$ & $36.234(90,7)$ & $1,25(1,20-1,30)$ & $<0,001$ \\
\hline Sem esc./Fund. I & $390(0,3)$ & $52(13,3)$ & $338(86,7)$ & $1,88(1,40-2,52)$ & $<0,001$ \\
\hline
\end{tabular}




\begin{tabular}{|c|c|c|c|c|c|}
\hline \multicolumn{6}{|l|}{ Estado civil** } \\
\hline Casada/União estável & $61.583(46,2)$ & $4674(7,6)$ & $56.909(92,4)$ & 1 & \\
\hline Solteira & $69.370(52,1)$ & $5.855(8,4)$ & $63.515(91,6)$ & $1,12(1,08-1,17)$ & $<0,001$ \\
\hline Viúva & $340(0,3)$ & $37(10,9)$ & $303(89,1)$ & $1,49(1,06-2,09)$ & 0,023 \\
\hline Separada jud./Div. & $1975(1,5)$ & $196(9,9)$ & $1779(90,1)$ & $1,34(1,15-1,56)$ & $<0,001$ \\
\hline \multicolumn{6}{|l|}{ Ocupação } \\
\hline Outra & $79.330(59,1)$ & $6.222(7,8)$ & $73.108(92,2)$ & 1 & \\
\hline Do lar & $54.992(40,9)$ & $4.657(8,5)$ & $50.335(91,5)$ & $1,09(1,05-1,13)$ & $<0,001$ \\
\hline \multicolumn{6}{|l|}{ Consultas pré-natais } \\
\hline 7 ou mais & $95.146(71,3)$ & $5.415(5,7)$ & $89.731(94,3)$ & 1 & \\
\hline 1 a 6 & $35.137(26,3)$ & $4.748(13,5)$ & $30.389(86,5)$ & $2,59(2,49-2,70)$ & $<0,001$ \\
\hline Nenhuma & $3.244(2,4)$ & $585(18,0)$ & $2.659(82,0)$ & $3,65(3,32-4,00)$ & $<0,001$ \\
\hline \multicolumn{6}{|l|}{ Tipo de parto } \\
\hline Vaginal & $54.157(40,3)$ & $4.363(8,1)$ & $49.794(91,9)$ & 1 & \\
\hline Cesáreo & $80.156(59,7)$ & $6.513(8,1)$ & $73.643(91,9)$ & $1,01(0,98-1,05)$ & 0,648 \\
\hline \multicolumn{6}{|l|}{ Local do nascimento } \\
\hline Hospital & $133.889(99,7)$ & $10.775(8,0)$ & $123.114(92,0)$ & 1 & \\
\hline Outro & $429(0,3)$ & $102(23,8)$ & $327(76,2)$ & $3,56(2,85-4,46)$ & $<0,001$ \\
\hline \multicolumn{6}{|l|}{ Idade gestacional*** } \\
\hline A termo $(\geq 37)$ & $117.299(89,2)$ & $4.340(3,7)$ & $112.959(96,3)$ & 1 & \\
\hline Pré-termo (<37) & $14.217(10,8)$ & $6.249(44,0)$ & $7.968(56,0)$ & $20,41(19,52-21,35)$ & $<0,001$ \\
\hline \multicolumn{6}{|l|}{ Sexo } \\
\hline Masculino & $68.774(51,2)$ & $5.095(7,4)$ & $63.679(92,6)$ & 1 & \\
\hline Feminino & $65.536(48,8)$ & $5.775(8,8)$ & $59.761(91,2)$ & $1,21(1,16-1,26)$ & $<0,001$ \\
\hline \multicolumn{6}{|l|}{ Cor da pele } \\
\hline Branca & $112.785(84,7)$ & $8.921(7,9)$ & $103.864(92,1)$ & 1 & \\
\hline Preta & $8.821(6,6)$ & $835(9,5)$ & $7.986(90,5)$ & $1,22(1,13-1,31)$ & $<0,001$ \\
\hline Amarela & $112(0,1)$ & $10(8,9)$ & $102(91,1)$ & $1,14(0,60-2,19)$ & 0,690 \\
\hline Parda & $10.891(8,2)$ & $951(8,7)$ & $9.940(91,3)$ & $1,11(1,04-1,96)$ & 0,003 \\
\hline Indígena & $578(0,4)$ & $49(8,5)$ & $529(91,5)$ & $1,08(0,80-1,45)$ & 0,614 \\
\hline \multicolumn{6}{|l|}{ Anomalia } \\
\hline Sem & $129.285(99,0)$ & $10.154(1,0)$ & $119.131(92,1)$ & 1 & \\
\hline Com & $1.307(1,0)$ & $304(23,3)$ & $1.003(76,7)$ & $3,56(3,12-4,05)$ & $<0,001$ \\
\hline Total & $134.322(100,0)$ & $10.879(8,1)$ & $123.442(91,9)$ & & \\
\hline
\end{tabular}

Constatou-se que a maior força de associação foi entre o BPN e a idade gestacional inferior a 37 semanas, indicando que os NV prematuros apresentaram 20,4 vezes a chance de apresentar baixo peso quando comparados aos nascidos a termo, seguida da ausência de consulta pré-natal. Isso denota que um NV de uma mãe que não fez nenhuma consulta pré-natal apresentou 3,65 vezes a chance de nascer com BPN comparado a um NV cuja mãe realizou sete ou mais consultas. Após o ajuste univariado, foram consideradas para a análise de regressão logística múltipla as variáveis apresentadas na Tabela 2 para o Modelo 1. 
Tabela 2 - Resultados da análise de regressão logística múltipla para os NV, tendo como desfecho o BPN, SINASC/RS, 2011 - Modelo 1

\begin{tabular}{|c|c|c|c|c|}
\hline Variáveis independentes & $\beta_{i}$ & $(\text { Wald })^{2 *}$ & OR Ajustado (IC 95\%) & p-valor \\
\hline \multicolumn{5}{|l|}{ Idade materna (anos) } \\
\hline 20 a 34 & & 66,68 & 1 & \\
\hline$\geq 35$ & 0,231 & 41,98 & $1,26(1,18-1,35)$ & $<0,001$ \\
\hline$<20$ & $-0,169$ & 20,54 & $0,84(0,78-0,91)$ & $<0,001$ \\
\hline \multicolumn{5}{|l|}{ Paridade } \\
\hline 1 a 2 & & 229,99 & 1 & \\
\hline Nulípara & 0,418 & 212,12 & $1,52(1,44-1,61)$ & $<0,001$ \\
\hline$\geq 3$ & $-0,027$ & 0,47 & $0,98(0,90-1,05)$ & 0,495 \\
\hline \multicolumn{5}{|l|}{ Escolaridade } \\
\hline Superior Incompleto/Completo & & 37,17 & 1 & \\
\hline Fundamental II/Ensino Médio & 0,172 & 34,99 & $1,19(1,12-1,26)$ & $<0,001$ \\
\hline Sem escolaridade/Fundamental I & 0,370 & 3,81 & $1,45(0,99-2,10)$ & 0,051 \\
\hline \multicolumn{5}{|l|}{ Estado civil } \\
\hline Casada/União estável & & 7,83 & 1 & \\
\hline Solteira & $-0,009$ & 0,11 & $0,99(0,94-1,04)$ & 0,736 \\
\hline Viúva & 0,372 & 2,99 & $1,45(0,95-2,21)$ & 0,084 \\
\hline Separada jud./Div. & 0,200 & 4,50 & $1,22(1,01-1,47)$ & 0,034 \\
\hline \multicolumn{5}{|l|}{ Ocupação } \\
\hline Outra & & & 1 & \\
\hline Do lar & $-0,070$ & 6,84 & $0,93(0,88-0,98)$ & 0,003 \\
\hline \multicolumn{5}{|l|}{ Consultas pré-natais } \\
\hline 7 ou mais & & 663,57 & 1 & \\
\hline 1 a 6 & 0,618 & 523,95 & $1,86(1,76-1,96)$ & $<0,001$ \\
\hline Nenhuma & 1,142 & 254,57 & $3,13(2,72-3,61)$ & $<0,001$ \\
\hline \multicolumn{5}{|l|}{ Local do nascimento } \\
\hline Hospital & & & 1 & \\
\hline Outro & 1,013 & 37,93 & $2,75(2,00-3,80)$ & $<0,001$ \\
\hline \multicolumn{5}{|l|}{ Idade gestacional (semanas) } \\
\hline A termo $(\geq 37)$ & & & 1 & \\
\hline Pré-termo $(<37)$ & 2,930 & 13839,23 & $18,74(17,84-19,67)$ & $<0,001$ \\
\hline \multicolumn{5}{|l|}{ Sexo } \\
\hline Masculino & & & 1 & \\
\hline Feminino & 0,289 & 138,54 & $1,34(1,27-1,40)$ & $<0,001$ \\
\hline \multicolumn{5}{|l|}{ Anomalia } \\
\hline Sem & & & 1 & \\
\hline Com & 0,948 & 113,37 & $2,58(2,17-3,07)$ & $<0,001$ \\
\hline
\end{tabular}

O valor de -2 In da verossimilhança para o Modelo 1 foi $49.144,149$. Avaliando o valor da deviance deste modelo incluindo a cor da pele em relação ao modelo sem a variável $(-2$ In da verossimilhança $=49.394,481)$, tem-se $G=250,332$ que se mostrou superior ao valor de $\chi_{5 \%, 1}^{2}=3,84$, indicando que a cor da pele deve permanecer no Modelo 1, embora não tenha se mostrado significativa na análise univariada. 
Na análise multivariável observou-se que, depois dos pré-termos $(\mathrm{OR}=18,74)$, o maior fator de risco foi a ausência de consulta pré-natal $(O R=3,13)$, o fato de não nascer no hospital $(\mathrm{OR}=2,75)$ e da presença de anomalia congênita $(\mathrm{OR}=2,58)$. Um modelo alternativo foi proposto (Modelo 2) pois, na análise univariada, o tipo de parto não foi significativo (p-valor=0,64). Entretanto, foi significativa quando avaliado junto com as variáveis do Modelo 1. Os resultados são apresentados na Tabela 3.

Tabela 3 - Análise de regressão logística múltipla dos NV, tendo como desfecho o BPN, SINASC/RS, 2011 - Modelo 2

\begin{tabular}{|c|c|c|c|c|}
\hline Variáveis independentes & $\beta_{i}$ & $(\text { Wald })^{2^{*}}$ & OR Ajustado (IC 95\%) & p-valor \\
\hline \multicolumn{5}{|l|}{ Idade materna (anos) } \\
\hline 20 a 34 & & 56,57 & 1 & \\
\hline$\geq 35$ & 0,217 & 36,90 & $1,24(1,16-1,33)$ & $<0,001$ \\
\hline$<20$ & $-0,153$ & 16,62 & $0,86(0,80-0,92)$ & $<0,001$ \\
\hline \multicolumn{5}{|l|}{ Paridade } \\
\hline 1 a 2 & & 218,83 & 1 & \\
\hline Nulípara & 0,411 & 204,06 & $1,51(1,43-1,60)$ & $<0,001$ \\
\hline$\geq 3$ & $-0,017$ & 0,18 & $0,98(0,91-1,06)$ & 0,668 \\
\hline \multicolumn{5}{|l|}{ Escolaridade } \\
\hline Superior Incompleto/Completo & & 42,04 & 1 & \\
\hline Fundamental II/Ensino Médio & 0,184 & 39,79 & $1,20(1,14-1,27)$ & $<0,001$ \\
\hline Sem escolaridade/Fundamental I & 0,382 & 4,05 & $1,47(1,01-2,13)$ & 0,044 \\
\hline \multicolumn{5}{|l|}{ Estado civil } \\
\hline Casada/União estável & & 7,38 & 1 & \\
\hline Solteira & $-0,001$ & 0,00 & $0,99(0,95-1,05)$ & 0,981 \\
\hline Viúva & 0,375 & 3,05 & $1,46(0,96-2,22)$ & 0,081 \\
\hline Separada jud./Div. & 0,197 & 4,34 & $1,22(1,01-1,47)$ & 0,037 \\
\hline \multicolumn{5}{|l|}{ Ocupação } \\
\hline Outra & & & 1 & \\
\hline Do lar & $-0,070$ & 6,84 & $0,93(0,88-0,98)$ & 0,009 \\
\hline \multicolumn{5}{|l|}{ Consultas pré-natais } \\
\hline 7 e mais & & 681,68 & 1 & \\
\hline 1 a 6 & 0,633 & 540,48 & $1,88(1,79-1,99)$ & $<0,001$ \\
\hline Nenhuma & 1,162 & 263,01 & $3,20(2,78-3,68)$ & $<0,001$ \\
\hline \multicolumn{5}{|l|}{ Tipo de parto } \\
\hline Vaginal & & & 1 & \\
\hline Cesáreo & 0,120 & 20,24 & $1,13(1,07-1,19)$ & $<0,001$ \\
\hline \multicolumn{5}{|l|}{ Local do nascimento } \\
\hline Hospital & & & 1 & \\
\hline Outro & 1,066 & 41,78 & $2,91(2,10-4,01)$ & $<0,001$ \\
\hline \multicolumn{5}{|l|}{ Idade gestacional (semanas) } \\
\hline A termo $(\geq 37)$ & & & 1 & \\
\hline Pré-termo $(<37)$ & 2,927 & 13792,03 & $18,67(17,78-19,60)$ & $<0,001$ \\
\hline \multicolumn{5}{|l|}{ Sexo } \\
\hline Masculino & & & 1 & \\
\hline Feminino & 0,291 & 139,84 & $1,34(1,27-1,40)$ & $<0,001$ \\
\hline
\end{tabular}




\begin{tabular}{lllll}
\hline Anomalia & & & & \\
\hline Sem & & & 1 & \\
Com & 0,941 & 111,71 & $2,56(2,15-3,05)$ & $<0,001$ \\
\hline *Estatística resultante do teste de Wald & & & &
\end{tabular}

*Estatística resultante do teste de Wald

Para indicar o melhor modelo, com ou sem a covariável tipo de parto utilizouse o teste da razão de verossimilhança. Para a realização deste teste, primeiramente, foram consideradas todas as covariáveis e, para a comparação entre os modelos ajustados, foi verificado o efeito da inclusão de cada covariável, ou seja, se esta melhorava o poder de explicação do modelo. Assim, o valor da deviance encontrado foi $G=49394,481-49372,287=22,194$, sendo este valor superior ao $\chi_{5 \%, 1}^{2}=3,84$, indicando a importância do tipo de parto no modelo. Analisando-se os dois modelos propostos foi possível, sem considerar o tipo de parto (Modelo 1), estimar a probabilidade de um bebê apresentar BPN, dada por:

$$
P(y \mid x)=\frac{1}{1+e^{-(\ln o d d s)}}=\frac{1}{1+e^{-(3,855)}}=0,979
$$

Considerando-se o Modelo 2, com o tipo de parto, a probabilidade de um bebê apresentar BPN, considerando todos os fatores de risco significativos foi $98,7 \%$, dada por:

$$
P(y \mid x)=\frac{1}{1+e^{-(\operatorname{lnodds})}}=\frac{1}{1+e^{-(4,352)}}=0,987
$$

Para os testes de Hosmer-Lemeshow observou-se um melhor ajuste para o Modelo 2 ( $p=0,06)$, com um percentual de classificação global dos eventos considerados igual a 92,5\%, indicando uma boa capacidade de predição do modelo. Verificou-se que não há evidências de observações influentes, pois os valores encontrados para a distância de Cook foram < 1 e não foram notadas observações discrepantes ou outliers. Assim, o Modelo 2 foi selecionado para explicar o BPN em função das covariáveis estudadas. 


\section{DISCUSSÃO}

Segundo o relatório Situação Mundial da Infância 2013, do Fundo das Nações Unidas para a Infância (UNICEF, 2013a), 15\% das crianças nasceram no mundo com baixo peso entre 2007 e 2011. O percentual tem se mantido estável desde 2000, em torno de $8 \%$ do total de nascimentos no Brasil, sendo que esta prevalência evoluiu de 7,7\% em 2000 para 8,4\% em 2011. As regiões com as maiores prevalências são a Sudeste e a Sul e, as menores, a Norte e a Nordeste. Os percentuais das primeiras não são compatíveis com o nível de desenvolvimento dessas regiões, nem mesmo com os avanços dos indicadores de saúde materno-infantil (UNICEF, 2013b). Observou-se o fenômeno descrito como o "paradoxo do baixo peso ao nascer" (SILVA; SILVA; BARBIERI; BETTIOL; CARVALHO; RIBEIRO; et al., 2010), onde a prevalência é mais elevada nas regiões mais desenvolvidas do país, em comparação com aquelas de menor desenvolvimento. As causas para tal paradoxo ainda precisam ser melhores investigadas, mas algumas são descritas na literatura, como a alta utilização de técnicas de reprodução assistida e o número excessivo de cesarianas (MATIJASEVICH; SILVEIRA; MATOS; RABELLO; FERNANDES; MARANHÃO; et al., 2013). Esta desigualdade na distribuição espacial do BPN reforça a necessidade de maiores investimentos na melhoria da rede de assistência médica e sua utilização por toda população para diminuir sua ocorrência.

Os fatores como raça, etnia e a idade da mãe também influenciam na prevalência dos casos de BPN, segundo o UNICEF (2013c). As mulheres negras respondem pelo maior percentual de nascimentos de crianças abaixo do peso $(9,4 \%)$, seguida pelas brancas $(8,3 \%)$ e pardas $(8,2 \%)$, sendo os menores valores entre mulheres amarelas $(7,6 \%)$ e indígenas (7,7\%). A raça do RN é uma variável que não pode ser modificada. No entanto, os sistemas sociais e de saúde devem estar atentos às situações que podem gerar maiores riscos à ocorrência de BPN naquelas mais vulneráveis ao problema.

Em relação à idade materna, encontrou-se maior risco de BPN entre aquelas com $\geq 35$ anos $(O R=1,24)$, e um efeito protetor entre as adolescentes $(O R=0,86)$. Guimarães e Velasquez-Melendez (2002), em estudo realizado em Itaúna-MG, de 1997 a 2000, 
mostraram que os filhos de mulheres com mais de 35 anos apresentaram maior risco de BPN $(O R=1,70)$, seguido pelas mães na faixa entre 10 e 19 anos $(O R=1,59)$. Segundo Silva (2003), as gestações em mulheres com mais idade estão aumentando, visto que estas adiam a gestação para priorizar a carreira profissional, buscando estabilidade econômica e parceiro estável. Outro aspecto é a maior probabilidade do surgimento de patologias ou intercorrências médicas associadas ao avanço da idade materna. Horon, Strobino e MacDonald (1983) citam como intercorrências na gravidez mais frequentes para essa faixa etária o diabetes e a hipertensão arterial, que modificam o intercurso da gestação e aumentam a ocorrência de partos prematuros. Em estudo epidemiológico transversal, em hospital do Rio de Janeiro, identificou a idade materna como um fator de risco $(O R=1,123)$, sendo que, a cada ano a mais havia um aumento de $12,3 \%$ no risco de BPN (CAPELLI; PONTES; PEREIRA; SILVA; CARMO; BOCCOLINI; et al., 2014).

Roth, Hendrickson e Stowell (1998) citam que, dentre os mecanismos explicativos, para a ocorrência de BPN em mulheres com idade $<20$ anos, encontramse os de natureza biológica, como imaturidade do sistema reprodutivo, ganho de peso inadequado durante a gestação e fatores socioculturais, como pobreza e marginalidade social, combinados ao estilo de vida adotado pela adolescente. Embora haja relevância de ambos os motivos, a falta de cuidados pré-natais das adolescentes, associada à pobreza e níveis baixos de instrução, são os principais fatores na cadeia causal do BPN. Para Maia (1997), a maior parte das adolescentes grávidas pertence a estratos sociais menos favorecidos, sem acesso às informações necessárias durante a gestação e sem adequada assistência pré-natal. Essa associação pode estar relacionada ao baixo padrão socioeconômico, pois a escolaridade materna pode ser vista como um indicador de condição social, onde o maior grau de instrução facilita o acesso a emprego e melhoria da situação financeira da família. A idade materna não é uma variável em que se possa atuar diretamente, mas as intensificações nas ações de educação em saúde e nos programas que envolvem planejamento familiar podem contribuir para reduzir o BPN. Ainda, o sistema de saúde deve fornecer uma assistência periódica e de qualidade. 
Observou-se que o risco de BPN foi maior $(\mathrm{OR}=1,51)$ entre as mulheres nulíparas, sendo que, em São Paulo, de 2002 e 2003, em estudo com 388.980 NV, Minuci e Almeida (2009), encontráramos autores relatam $O R=1,44$, enquanto que Rojas, Carminatti, Hafemann, Stein e Francisco (2013), em estudo com puérperas de recém-nascidos com baixo peso em São José-SC, de 2010 e 2011, encontraram OR=2,45. Neste sentido, Silva, Barbieri, Gomes e Bettiol (1998) sugerem que a maior frequência de BPN em mulheres nulíparas está associada a gestações de pré-termo, enquanto que Costa e Gotlieb (1998) descrevem que as primíparas apresentam maior presença de BPN atribuídas à sua menor idade.

A escolaridade materna se mostrou como fator de risco para aquelas que não tinham Curso Superior, completo ou incompleto. Segundo Maia e Souza (2010), mães com baixa escolaridade têm acesso dificultado a serviços de pré-natal de melhor qualidade e as maternidades melhor equipadas. Viana, Taddei, Cocetti e Warkentin (2013), observaram maiores proporções de BPN entre filhos de mulheres com escolaridade inferior a quatro anos, com $O R=2,19$, com dados da Pesquisa Nacional de Demografia e Saúde da Criança e da Mulher (PNDS). Para o presente estudo, o fator de risco compreendeu mães com ensino fundamental I| ou ensino médio. Para Nascimento (2003), as mães com até oito anos de estudo apresentaram maiores chances de gerar crianças com BPN, possivelmente devido à desinformação e por ter menor interesse ou maior dificuldade de acesso a serviços de saúde. Este fato pode ser esclarecido por estas mães terem um padrão socioeconômico desfavorável, o que provavelmente ocasionará em um menor ganho de peso na gestação e um início mais tardio do pré-natal. A escolaridade materna sobre a saúde materno-infantil é uma relação de grande importância, que pode ser entendida pelo fato de mulheres com maior nível de instrução são mais hábeis em cuidar de si mesmas, apresentam maior conhecimento dos cuidados que devem ser realizados e têm uma condição socioeconômica diferenciada. Pesquisas desenvolvidas em diferentes países evidenciam que a educação é o mais forte preditor socioeconômico, de forma isolada, em relação às condições de saúde e o mais importante fator determinante do peso ao nascer de uma população (HOWARD; MARSHALL; KAUFMAN; SAVITZ, 2006). 
Verificou-se que o fato da mãe ser separada $(O R=1,22)$ foi fator de risco significativo para BPN. Para Victora, Barros e Vaughan (1989) e Stusser, Paz, Ortega, Pineda, Infante, Martin e Ordónez (1993), o fato da mãe ser solteira é um fator importante a ser considerado, pois além da desvantagem psicológica, a ausência do pai, em geral, traz menor estabilidade econômica para a família, podendo se constituir em fator de risco para BPN. Coimbra, Silva, Mochel, Alves, Ribeiro, Aragão et al. (2003), constataram que a ausência de companheiro influencia a adesão das gestantes à assistência pré-natal. Caso não haja adesão, o monitoramento dos efeitos indesejáveis à mãe e ao RN ficaria comprometido. A falta da figura paterna em geral pode trazer menor estabilidade financeira para a família, podendo constituir em fator de risco para o BPN. Em estudo da tendência secular do peso ao nascer em São Paulo, Monteiro, Benicio e Ortiz (2000), detectaram que o risco relativo de BPN aumentava para mães que viviam sem companheiro.

Foi observado efeito protetor e significativo $(\mathrm{OR}=0,93)$ para crianças nascidas de mulheres que não exerciam ocupações formais externas ao lar. Este dado contradiz o resultado encontrado em Moraes, Zanini, Riboldi e Giugliani (2012) com fator de risco para o BPN (OR=1,15). Já para Rojas, Carminatti, Hafemann, Stein e Francisco (2013), não foi encontrada associação significativa entre a variável ocupação da mãe e o BPN. Mariotoni e Barros Filho (2000) constataram que o trabalho materno fora de casa não exerceu efeito sobre o peso ao nascer em Campinas-SP. Maia e Souza (2010) constataram um aumento estatisticamente significante da prevalência de BPN em crianças cujas mães não trabalhavam. A proteção contra o BPN, conferida pelo trabalho materno fora do lar, possivelmente está ligada ao fator socioeconômico. Relacionado a isso, Monteiro e Freitas (2000) apontam que o acesso a alimentação e aos serviços essenciais, bem como a qualidade do ambiente são fatores que dependem do poder aquisitivo.

As crianças cujas mães não realizaram consulta pré-natal tiveram risco elevado e significativo $(O R=3,20)$ para $B P N$, sendo que, para aquelas que realizaram de uma a seis consultas, o risco foi OR=1,88. De acordo com Minuci e Almeida (2009) e Geib, Fréu, Brandão e Nunes (2010) um número insuficiente de consultas pré-natal constitui fator 
de risco para BPN. Isto também foi observado por Almeida, Costa, Gama, Amaral e Vieira (2014) em estudo no Nordeste brasileiro e por Pedraza, Rocha e Cardoso (2013), em Queimadas-PA, reforçando que a ausência ou a deficiência da atenção pré-natal estão associadas ao BPN. Entretanto, este efeito não foi confirmado por Guimarães e Velasquez-Melendez (2002), com crianças nascidas em hospitais de Itaúna-MG. Para Ribeiro, Guimarães, Lima, Sarinho e Coutinho (2009) e Surita, Suarez, Siani e Silva (2011), o número reduzido de consultas pode ser reflexo da dificuldade de acesso, relacionado a aspectos pessoais, sociais e econômicos, ou da má-qualidade nos serviços. Quanto maior o número de consultas pré-natais, menor o risco de a criança nascer com BPN (NASCIMENTO; GOTLIEB, 2001; CARNIEL; ZANOLLI; ANTÔNIO; MORCILLO, 2008). Conforme Almeida, Alencar, Schoeps, Novaes, Campbell e Rodrigues (2011), a preocupação não deve se limitar apenas ao número de consultas, durante a gravidez, mas também à qualidade da atenção pré-natal, uma vez que a adequada assistência possibilita identificar precocemente as gestações de risco, prevenindo o BPN. Assim, ressalte-se a importância de que o pré-natal seja iniciado o quanto antes, tenha a periodicidade correta e seja oferecido com qualidade.

O parto cesáreo se mostrou como fator de risco neste estudo $(O R=1,13)$, resultado concordante com Santos, Costa, Amaral, Vieira, Bacelar e Almeida (2014), em Feira de Santana-BA, de 2006 a 2012 (OR=1,34). Corroborando com estes achados há os estudos de Carniel, Zanolli, Antônio e Morcillo (2008) e Moraes, Zanini, Giugliani e Riboldi (2011), do mesmo modo Silva, Silva, Barbieri, Bettiol, Carvalho, Ribeiro e et al. (2010), após análise de nascimentos nas cidades de Ribeirão Preto-SP, e São Luís-MA, sugerem que isso ocorre, pois, o Brasil está passando por uma transição epidemiológica perinatal, com BPN mais prevalente nas regiões mais ricas, devido não só aos avanços na medicina neonatal, como também na redução do período de gestação, devido aos partos cesáreos eletivos. Segundo Carniel, Zanolli e Morcillo (2007) há mais cesarianas entre grupos de baixo risco obstétrico e de mulheres com melhores condições sociais, sugerindo que os critérios para indicação desse procedimento não são exclusivamente técnicos. Em contradição aos estudos, Rojas, 
Carminatti, Hafemann, Stein e Francisco (2013) identificaram que a cesariana não esteve relacionada significativamente com o BPN $(p=0,808)$.

Entre os bebês nascidos em outros locais, o risco foi de 2,91 vezes o dos que nasceram em um hospital. Este resultado foi reforçado em Moraes, Zanini, Riboldi e Giugliani (2012), no qual este risco encontrado foi de 2,16 vezes. Silva, Almeida, Ortiz, Alencar, Alencar, Schoeps, et al. (2009) chama atenção sobre a alta frequência de características negativas dos nascimentos domiciliares, tais como elevada proporção de mães de baixa escolaridade, sem companheiro e com assistência pré-natal deficiente. Isso pode sugerir dificuldades de acesso a serviços de atenção pré-natal e ao parto. A ocorrência desses partos pode ser entendida como evento sentinela para os serviços de saúde, indicando a importância da referência hospitalar para realização do parto antes mesmo do final da gestação. Mesmo que o risco de BPN seja maior em bebês que nasceram fora do hospital, a avaliação da qualidade da assistência hospitalar também merece aprofundamento, uma vez que este é o local predominante dos partos.

Constatou-se que a ocorrência do BPN esteve fortemente associada com a prematuridade $(O R=18,67)$, indicando que as crianças prematuras apresentaram risco significativamente superior de BPN quando comparadas com as nascidas a termo. Este resultado corrobora com o estudo de Almeida, Costa, Gama, Amaral e Vieira (2014), no qual encontraram $O R=21,0$. Conforme Maia e Souza (2010), a adequada assistência durante a gravidez é de importância fundamental pois possibilita a detecção e o tratamento de hábitos e condições maternas que podem contribuir para a ocorrência de partos prematuros.

Com relação ao sexo do RN, observou-se uma maior proporção de BPN entre as meninas (8,8\%). Para Viana, Taddei, Cocetti e Warkentin (2013) o sexo feminino apresentou $O R=1,55$, valor próximo do encontrado no presente estudo $(O R=1,34)$. $\mathrm{Na}$ literatura, não há um consenso se o sexo do bebê influencia o peso de nascimento, no entanto, sabe-se que as meninas apresentam menor peso que os meninos de mesma idade gestacional, com maior risco de restrição de crescimento intrauterino (KRAMER, 1987; MAIA; SOUZA, 2010; COSTA; GOTLIEB, 1998). Apesar dessa constatação e dos 
achados nos diversos estudos, o sexo do RN é uma variável em que não há possibilidade de intervenção.

A ocorrência de anomalia evidenciou fator de risco associado ao BPN $(O R=2,56)$. Comparando-se os resultados com os encontrados por Moraes, Zanini, Riboldi e Giugliani (2012), observou-se que a presença de anomalia congênita foi significativa no modelo proposto $(\mathrm{OR}=2,54)$. Mesmo que não seja tarefa fácil controlar a incidência de malformações congênitas em uma população, os hospitais públicos, locais que dão assistência perinatal à maioria da população de risco para o BPN, devem ser priorizados com melhorias na qualidade de assistência à gestante e ao RN através da capacitação de pessoal médico e da disponibilidade de tecnologia avançada em unidades de cuidados intensivos neonatais para os bebês que apresentam malformações congênitas.

\section{CONCLUSÃO}

Neste artigo foi realizado um estudo para identificar e quantificar os fatores de risco associados a crianças que nasceram com baixo peso no Rio Grande do Sul. Concluiu-se que, no modelo selecionado, os principais fatores de risco independentes associados ao BPN foram: a idade da mãe ( $\geq 35)$; mães nulíparas; sem Curso Superior; separadas judicialmente ou divorciadas; com seis ou menos consultas durante a gestação; que realizaram parto cesáreo; bebês que nasceram fora do hospital; prematuros; do sexo feminino e com anomalia congênita. O fator de risco mais importante para BPN foi a idade gestacional, logo salienta-se que o pré-natal é de extrema importância para as futuras mamães, pois é através dele que alterações são detectadas e tratadas a tempo, evitando-se, assim, problemas para a saúde da mãe e do bebê.. Assim, considerando os presentes achados, aliados aqueles encontrados na literatura, destaca-se a importância da implementação de medidas voltadas para a saúde da gestante e do bebê, para a redução da incidência do BPN. Recomenda-se também, que sejam consideradas ações efetivas aos grupos vulneráveis, no sentido de redução das taxas de BPN, tendo em vista a forte associação desta característica com as taxas de mortalidade infantil. 


\section{REFERÊNCIAS}

ALMEIDA AHV, COSTA MCO, GAMA SGN, AMARAL MTR, VIEIRA GO. Low weight at birth in adolescent and young adult mothers in the Northeast Region of Brazil. Rev. Bras. Saúde Mater. Infant. 2014;14(3):279-286.

ALMEIDA MF, ALENCAR GP, SCHOEPS D, NOVAES HMD, CAMPBELL O, RODRIGUES LC. Sobrevida e fatores de risco para mortalidade neonatal em uma coorte de nascidos vivos de muito baixo peso ao nascer, na Região Sul do Município de São Paulo, Brasil. Cad. Saúde Pública. 2011;27(6):1088-1098.

CAPELLI JCS, PONTES JS, PEREIRA SEA, SILVA AAM, CARMO CN, BOCCOLINI CS, et al. Birth weight and factors associated with the prenatal period: a cross-sectional study in a maternity hospital of reference. Ciência. Saúde Coletiva. 2014;19(7):2063-2072.

CARNIEL EF, ZANOLLI ML, ANTÔNIO MARGM, MORCILLO AM.Determinantes do baixo peso ao nascer a partir das declarações de nascidos vivos. Rev. Bras. Epidemiol. 2008;11(1):169-179.

CARNIEL EF, ZANOLLI ML, MORCILLO AM. Risk factors for the indication of caesarean section in Campinas (SP). Rev. Bras. Ginecol. Obstet. 2007; 29: 34-40.

COIMBRA LC, SILVA AAM, MOCHEL EG, ALVES MTSSB, RIBEIRO VS, ARAGÃO VMF et al. Fatores associados à inadequação do uso da assistência pré-natal. Rev. Saúde Pública. 2003;37(4):456-462.

COSTA CE, GOTLIEB SLD. Estudo epidemiológico do peso ao nascer a partir da Declaração de Nascido Vivo. Rev. Saúde Pública. 1998;32(4):328-334.

GEIB LTC, FRÉU CM, BRANDÃO M, NUNES ML. Social and biological determinants of infant mortality in population cohort in the city of Passo Fundo, Rio Grande do Sul State. Ciênc. Saúde Coletiva. 2010;15(2):363-370.

GUIMARÃES EAA, VELASQUEZ-MELENDEZ G. Low birth weight determinants from the Born Alive National Surveillance System in Itaúna, Minas Gerais. Rev. Bras. Saúde Mater. Infant. 2002;2(3):283-290.

HARRISON GA, WEINER JS, TANNER JM. Biologia humana: introdução à evolução, variação e crescimento humanos. São Paulo: Companhia Editora Nacional e Editora da Univ. São Paulo; 1971.

HORON IL, STROBINO DM, MACDONALD HM. Birth weights among infants born to adolescent and adult woman. Am J Obstet Gynecol. 1983;146(4):444-449.

HORTA BL, BARROS FC, HALPERN R, VICTORIA CG. Low birthweight in two populationbased cohorts in southern Brazil. Cad. Saúde Pública. 1996;12(1):27-31. 
HOSMER JDW, LEMESHOW S. Applied Logistic Regression. New York: John Wiley; 1989.

HOWARD DL, MARSHALL SS, KAUFMAN JS, SAVITZ DA. Variations in low birth weight and preterm delivery among blacks in relation to ancestry and nativity: New York City, 19982002. Pediatrics. 2006;118:1399-1405.

JEKEL JF, ELMORE JG, KATZ DL. Epidemiologia, bioestatística e medicina preventiva. Porto Alegre: Artmed; 1999.

KRAMER MS. Determinants of low birth weight: methodological assessment and metaanalysis. Bull World Health Organ. 1987;65(5):663-737.

MAIA MAC. Caracterização dos nascidos vivos hospitalares no primeiro ano de implantação do Subsistema de Informação sobre Nascidos Vivos, em município de Minas Gerais, Brasil, 1996. Rev. Saúde Pública. 1997;31:581-585.

MAIA RRP, SOUZA JMP. Factors associated with the low birth weight in municipality in Northern Brazil. Rev. Bras. Crescimento Desenvolv. Hum. 2010;20(3):736-744.

MARIOTONI GGB, BARROS FILHO AA. Peso ao nascer e características maternas ao longo de 25 anos na Maternidade de Campinas. J Pediatr. 2000;76(1):55-64.

MATIJASEVICH A, SILVEIRA MF, MATOS ACG, RABELLO ND, FERNANDES RM, MARANHÃO AG, et al. Estimativas corrigidas da prevalência de nascimentos pré-termo no Brasil, 2000 a 2011. Epidemiologia e Serviços de Saúde. 2013;22(4):557-564.

MCCORMICK MC. The contribution of low birth weight to infant mortality and childhood morbidity. New England Journal of Medicine. 1985;312:82-90.

MINISTÉRIO DA SAÚDE [Internet]. Sistema de Informações sobre Nascidos Vivos $\begin{array}{lllll}\text { SINASC } & \text { [cited } & 2015 & \text { sep } & 25]\end{array}$ from:portal.saude.gov.br/portal/saude/visualizar_texto.cfm?idtxt=21379.

MINUCI EG, ALMEIDA MF. Birth weight intra-urban differentials in the city of São Paulo. Rev. Saúde Pública. 2009;43(2):256-266.

MONTEIRO CA, BENICIO MHDA, ORTIZ LP. Secular trends in birth weight in S. Paulo city, Brazil (1976-1998). Rev. Saúde Pública. 2000;34(6):26-40.

MONTEIRO CA, FREITAS ICM. Evolução de condicionantes socioeconômicas da saúde na infância na cidade de São Paulo (1984-1996). Rev. Saúde Pública. 2000;34(6):8-12.

MORAES AB, ZANINI RR, GIUGLIANI ERJ, RIBOLDI J. Tendência da proporção de baixo peso ao nascer, no período de 1999-2004, por microrregião do Rio Grande do Sul, Brasil: uma análise multinível. Cad. Saúde Pública. 2011;27:229-240. 
MORAES AB, ZANINI RR, RIBOLDI J, GIUGLIANI ER. Risk factors for low birth weight in Rio Grande do Sul State, Brazil: classical and multilevel analysis. Cad. Saúde Pública. 2012;28(12):2293-2305.

NASCIMENTO LFC. Pregnants profile in prenatal and perinatal stages: comparative study between public and private health services in Guaratinguetá, São Paulo. Rev. Bras. Saúde Mater. Infant. 2003;3(2):187-194.

NASCIMENTO LFC, GOTLIEB SLD. Fatores de risco para o baixo peso ao nascer, com base em informações da declaração de nascido vivo em Guaratinguetá, SP, no ano de 1998. Inf Epidemiol. 2001;10(3):113-120.

PEDRAZA DF, ROCHA ACD, CARDOSO MVLML. Prenatal care and birth weight: an analysis in the context of family health basic units. Rev. Bras. Ginecol. Obstet. 2013;35(8):349-356.

RIBEIRO AM, GUIMARÃES MJ, LIMA MC, SARINHO SW, COUTINHO SB. Risk factors for neonatal mortality among children with low birth weight. Rev. Saúde Pública. 2009;43(2):246-255.

ROJAS PFB, CARMINATTI APS, HAFEMANN F, STEIN AC, FRANCISCO CC. Predictive maternal factors for low birth weight: a case-control study. Arq. Catarin. Med. 2013;42(1):68-75.

ROTH J, HENDRICKSON J, STOWELL DW. The risk of teen mothers having low birth weight babies: implications of recent medical research for school health personnel. JSch Health. 1998;68:271-275.

SANTOS NLAC, COSTA MCO, AMARAL MTR, VIEIRA GO, BACELAR EB, ALMEIDA AHV. Teenage pregnancy: analysis of risk factors for low birth weight, prematurity and cesarean delivery. Ciênc. Saúde Coletiva. 2014;19(3):719-726.

SILVA AA, BARBIERI MA, GOMES UA, BETTIOL $H$. Trends in low birth weight: a comparison of two birth cohorts separated by a 15-year interval in Ribeirão Preto, Brazil. Bull World Health Organ. 1998;76(1):73-84.

SILVA AAM, SILVA LM, BARBIERI MA, BETTIOL H, CARVALHO LM, RIBEIRO VS et al.The epidemiologic paradox of low birth weight in Brazil. Rev. Saúde Pública. 2010;44(5):767-775.

SILVA, TRSR. 0 peso ao nascer no município de Presidente Prudente, São Paulo de 1998 a 2001 [dissertation]. São Paulo: Universidade Federal de São Paulo/USP; 2003. 
SILVA ZP, ALMEIDA MF, ORTIZ LP, ALENCAR, ALENCAR AP, SCHOEPS $D$, et al. Características dos nascidos vivos, das mães e mortalidade neonatal precoce na Região Metropolitana de São Paulo, Brasil. Cad. Saúde Pública. 2009;25(9):1981-1989.

STUSSER R, PAZ G, ORTEGA M, PINEDA S, INFANTE O, MARTIN P, ORDONÉZ C. Riesgo de bajo peso al nacer en el área plaza de la Habana. Bol Ofic Sanit Panam. 1993;3:228240.

SURITA FGC, SUAREZ MBB, SIANI S, SILVA JLP. Factors associated with low birth weight among adolescents in the Brazil Southeast Region. Rev. Bras. Ginecol. Obstet. 2011;33(10):286-291.

TIAGO LF, CALDEIRA AP, VIEIRA MA. Risk factors for low birthweight in a public maternity of inner Minas Gerais State. Rev Pediatr (São Paulo). 2008;30(1):8-14.

UNICEF [Internet]. Brasília: Fundo das Nações Unidas para a Infância; 2013a [cited 2015 sep. 27].. Situação mundial da infância 2013. Available from: http://www.unicef.org/brazil/pt/PT_SOWC2013.pdf.

UNICEF [Internet]. Brasília: Fundo das Nações Unidas para a Infância; 2013b [cited 2015 sep 27]. Estudo faz alerta sobre a situação da prematuridade no Brasil. Available from: http://www.unicef.org/brazil/pt/media_25849.html.

UNICEF [Internet]. Brasília: Fundo das Nações Unidas para a Infância; 2013c [cited 2015 sep 27 Prematuridade e suas Possíveis Causas (Estudo). Available from: http://www.unicef.org/brazil/pt/br_prematuridade_possiveis_causas.pdf.

VIANA KJ, TADDEI JAAC, WARKENTIN S. Birth weight in Brazilian children under two years of age. Cad. Saúde Pública. 2013;29(2):349-356.

VICTORIA CG, BARROS FC, VAUGHAN J. Epidemiologia da desigualdade. São Paulo: Hucitec; 1989.

WORLD HEALTH ORGANIZATION [Internet]. Classifications [cited 2015 sep 16]. International statistical classification of diseases and reacted health problems, tenth revision. Available from:

http://www.who.int/classifications/icd/ICD10Volume2_en_2010.pdf. 